\title{
ANNALS \\ OF THE \\ RHEUMATIC DISEASES
}

\section{THE EVALUATION OF THE ROENTGENOLOGIC FINDINGS IN ARTHRITIS}

\author{
By JOHN D. CAMP
}

THE ideal or even generally accepted classification of the arthritides does not exist. The ones in vogue are all incomplete because the cause of the majority of arthritides is in doubt. Some classifications are based on the clinician's ideas of the cause - that is, infectious, chemical and traumatic. Some names for arthritis are merely clinical designations, with little or no specific meaning (for example, rheumatoid arthritis); other classifications are based on the dominant pathologic alteration in the joints (proliferative, degenerative), and still others on the dominant roentgenographic change (atrophic, hypertrophic, destructive).

Each of these classifications has its advantage and its field of usefulness. Each has distinct limitations and none is wholly satisfactory. The classification of the internist makes little sense to the roentgenologist, unfamiliar with the criteria supposedly necessary for a diagnosis of " allergic arthritis" or "metabolic arthritis." The schema of the pathologist seems academic to the general practitioner, who can only see the outside of the joint. The designations of the roentgenologist are open to criticism because several different conditions may produce more or less identical changes in joints. An adequate classification of articular disease, therefore, hard enough at best, cannot be made with regard to the roentgenographic or any other one feature alone, but only after a careful summation of all data, clinical, chemical, and roentgenographic.

Regardless of the clinical confusion, the roentgenologist can point out certain basic alterations which occur when the joints are diseased. In the early stage of arthritis a joint may be definitely, though moderately, diseased and yet the roentgenographic findings sometimes can be negative. There may be articular pain, definite tenderness, and even slight swelling, but no osteoporosis, lessening of articular space, alteration of bony contour, or increased periarticular density may be visible. The stage or degree of the articular disease is simply too early to alter the roentgenographic appearance. The disease is 
not subclinical, but it is "subroentgenographic." The only honest report that the roentgenologist can give, if he bases his diagnosis solely on the roentgenographic findings, is " negative joint." While making the roentgenogram he accidentally may have squeezed the patient's joints, and the patient's " ouch " may be considered sufficient evidence to indicate trouble; but the roentgenologist should not, in fairness to his science, borrow that bit of evidence to alter his report of what is in truth a normal-looking roentgenogram of a joint. For example, such " negative roentgenograms" are frequently encountered in cases of acute rheumatic fever, early acute gout, or serum sickness. In each case the joint may be red, tender, sometimes exquisitely so, and yet the swelling may not be sufficiently obvious in the roentgenogram to provoke comment.

The basic alterations that may be visible in roentgenograms in cases of arthritis are briefly as follows: Periarticular swelling, osteoporosis, diminution of interarticular (interosseous) space, and alterations in bony contour. The last-mentioned alteration may be either in the nature of proliferation or destruction of bone. Many of these are common to several different forms of arthritis. I shall comment on each of these basic alterations.

Periarticular swelling alone may be visible. It is revealed by an increased density of the soft tissue shadow around the joint or by an obvious increase in size of the periarticular shadow. Other alterations may be absent; when they are, the only diagnosis the roentgenologist has a right to make is " periarticular arthritis," or, better yet, "periarticular swelling." The roentgenographic evidence of synovial involvement is not always discrete, but synovial participation is suggested by increase in the density of the soft tissue nearest the intra-articular space.

The roentgenologic evidence of involvement of cartilage (destruction by invasion as in infectious, rheumatoid or atrophic arthritis or destruction by degeneration as in senescent or hypertrophic or osteoarthritis) is diminution of intra-articular (interosseous) space and irregularity of opposing surfaces.

The roentgenographic evidence of involvement of bone consists of osteoporosis, lipping of bone, marginal or subchondral proliferation of bone or destruction of bone (marginal or subchondral areas of erosion of bone).

Many different forms of arthritis may exhibit the same variety of these basic alterations as observed in roentgenograms. It is only because certain diseases of joints, which are fairly well recognised as entities, routinely present a more or less typical combination of these basic alterations that the roentgenologist can attach a name to the condition noted. A joint may be attacked by any one of a score or more of organisms. Its integrity may be violated in the course of 
gout, or its continuity altered as a result of a variety of injuries of different durations and degrees. It is too much to expect that each of these insults will cast a distinctive shadow, particularly when, with each condition, there are a variety of stages and degrees. There is too much similarity in the roentgenographic and even the pathologic response of joints to these various insults to permit the roentgenograms to be interpreted too dogmatically.

The reason for this is obvious if one studies the pathologic changes in the various types of arthritis. Regardless of the insulting agent, articular tissues can only react in one of a few ways. The synovial membrane responds to insult by inflammatory exudation (in one case [mild] with cells of a chronic reaction, in another case [severe] with cells of an acute type), by fibrosis and by villous proliferation. There is no distinguishing roentgenographic characteristic of any one form of synovitis. Articular cartilage reacts to all insults by variable degrees of disappearance (from inflammatory invasion in some cases, autolytic dissolution in certain cases of sepsis, degeneration in others). Except for suggestive data supplied by the speed and extent of destruction of cartilage - that is, in cases of septic, gonorrhœal, tuberculous or rheumatoid arthritis - there is no dependable roentgenographic differentiation of the various types of cartilage destruction. The bone reacts by varying degrees of proliferation and/or destruction-marginal or subchondral, or both.

Considering these fundamental limitations in pathologic and roentgenographic response, it would seem surprising that roentgenographic distinctions in joint disease can be made as often as they can. The reason the roentgenologist has any right to dogmatise and classify is that, in spite of a general similarity of roentgenographic change, there are combinations somewhat typical of the different disease entities. This can be illustrated by the following example used by Hench.

No matter with what object one hits the note middle C, on the piano, it always reacts by that note, $\mathrm{C}$ (and its overtones). Inherently, that note cannot react in any other way no matter whether one strikes it with a hammer, a pencil, a stick, or a finger. However, the tone may be different, and one may guess, from the tone, to what type of blow the tone is reacting. Likewise, in the roentgenographic study of arthritis, one must not expect massive differences, markedly characteristic alterations with each of the numerous possible insults, but one may rightly study the finer shades of differentiation, the "tone" of the roentgenographic changes, in order to suggest the etiologic type. It is by a study of subtle combinations of roentgenographic changes rather than discrete gross roentgenographic changes that one may be permitted to venture a clinical and not simply a roentgenographic diagnosis. Even then error stalks on every hand. 


\section{Classifications based on Roentgenographic Data}

Ignoring other classifications, the roentgenologist, using only roentgenologic evidence, would have to classify the arthritides as follows:

Periarthritis. - If there is periarticular thickening (increased density or an enlarged soft tissue shadow, or both), but no atrophy of bone, no loss of articular space, and no abnormality of bony contour, he must make a diagnosis of periarthritis. It might be an infectious periarthritis (the early stage of what later will progress to a definite intraarticular disease), or it might not be a true early arthritis, but really a periarticular fibrositis. The roentgenologist cannot distinguish between the periarthritis of rheumatic fever, of acute gout, of serum sickness, of early gonorrhœal arthritis, or early chronic or acute infectious (rheumatoid) arthritis, or of periarticular fibrositis, although the last-mentioned condition does not produce much real swelling.

Atrophic Arthritis. - If there is osteoporosis of the epiphyses and beginning of narrowing of the cartilage space, but no other abnormality of bony contour, the roentgenologist may recognise the changes as those of atrophic arthritis (clinical synonym: rheumatoid arthritis). There is probably some periarticular swelling. If there is a little marginal lipping of bone, instead of calling it atrophic and hypertrophic arthritis, he may discount the lipping and still call it atrophic arthritis. If there is considerable lipping of bone with atrophy of bone and loss of cartilage space, what should he call it? Atrophic and hypertrophic arthritis? Clinically the patient shouldn't have both, but roentgenologically he often has both. For example, an obese man with chronic infectious arthritis (atrophic or rheumatoid arthritis in the clinical sense) will often have typical atrophic changes in the hand and ankle, but in the ankle he also will have considerable proliferation of bone.

The roentgenologist cannot differentiate the atrophic arthritis seen in some cases of mild but moderately advanced gonorrhœeal arthritis from the atrophic arthritis of infectious (proliferative or rheumatoid) arthritis. If one has traumatic periarthritis (stiff, painful shoulder from injury, yet no alteration in cartilage or bone, it is not " arthritis ", but if the arm is immobilised long enough in a sling or at his side the roentgenogram will reveal osteoporosis in the shoulder-joint. How can this be differentiated from the early atrophic arthritis that is supposed to represent infectious (rheumatoid) arthritis ?

Hypertrophic Arthritis. - When the dominant feature is marginal proliferation of bone, the roentgenologist usually discounts the osteoporosis, destruction of cartilage, and swelling of soft tissue if present, and reports a hypertrophic arthritis. A descriptive report of destruction and hypertrophic changes is more informative and correct under such circumstances. 
Destructive Arthritis. - When there is not only destruction of cartilage but definite destruction of bone-that is, the destructive areas of bone and cartilage seem more dominant than the areas of overgrowth of bone-the roentgenologist usually calls it destructive arthritis, but without borrowing from the clinical data he cannot say whether it is the destructive arthritis of gonorrhcea or of severe rheumatoid arthritis or of severe osteo-arthritis as, for example, malum coxæ senilis.

\section{Limitations to Roentgenographic Classification}

There are certain objections to the use of the term "hypertrophic" arthritis as a clinical term. Hypertrophic changes (roentgenographic) are usually the dominant note of senescent arthritis (degenerative arthritis or osteo-arthritis). However, as in the case of the fat man with rheumatoid arthritis, there may be roentgenographic evidence of atrophic arthritis in the hands and some considerable atrophy of bone in the ankles, but the roentgenogram often will show considerable hypertrophic changes in the subastragaloid joint. Clinically, the case is one of rheumatoid arthritis (diffuse atrophic arthritis). If the roentgenologist sends the physician a report concerning the hand it will indicate atrophic arthritis in both the clinical and roentgenologic sense. If he sends a report concerning the foot (hypertrophic arthritis, with atrophy of bone), the physician may interpret the term hypertrophic arthritis, not in its roentgenologic sense, but in its clinical meaning, representing the syndrome variously called senescent arthritis, osteo-arthritis, and so forth. On the basis of such circumstances, papers have been written to suggest that clinical atrophic arthritis and hypertrophic arthritis often exist in the same case and may really be one disease. What was really present was clinical atrophic (rheumatoid) arthritis in all areas with roentgenologic evidence of atrophic arthritis in hands and roentgenologic evidence of atrophic plus hypertrophic arthritis in the ankle. One should not lose sight of the fact that atrophic changes are modified by weight in the weight-bearing joints and often show more hypertrophy than atrophy.

Unless one is careful to note associated atrophy of bone I do not see how the roentgenologist can consistently differentiate between the hypertrophic arthritis seen with clinical atrophic (rheumatoid) arthritis, the hypertrophic arthritis seen with gout or that seen in static arthritis (traumatic or postural arthritis). Psoriatic arthritis of the terminal phalangeal joints shows hypertrophy of bone. Primary osteo-arthritis also does, as do "baseball fingers." Typhoid arthritis of the spinal column is usually a hypertrophic arthritis, and even tuberculosis sometimes exhibits hypertrophic changes in the spinal column.

The trouble with the term "hypertrophic arthritis" is that the 
roentgenologist is merely describing the chief reaction seen in the roentgenogram, whereas the clinician, getting a report of hypertrophic arthritis, may think that the roentgenologist is diagnosing the syndrome "hypertrophic" arthritis (senescent arthritis or osteo-arthritis). That is one of the reasons why gout is so often missed. The clinician has been led to expect that gout should soon produce characteristic roentgenographic changes, and when the report comes back " hypertrophic arthritis" he promptly pigeon-holes it into the syndrome of osteoarthritis.

When the roentgenologist reports destructive arthritis, where is the clinician to put it ? "Destructive" arthritis is usually, I suppose, the late stage of severe rheumatoid (atrophic) arthritis, but it may be the end stage of gonorrhœal arthritis, severe psoriatic arthritis, tuberculous arthritis, septic arthritis, or of the severe arthritis that occurs in some cases of ulcerative colitis.

\section{Typical Roentgenograms of Various Types of Arthritis}

One should use the word " typical" instead of the terms " characteristic" and " diagnostic." When one looks from the clinical side to the roentgenographic aspect he finds certain typical changes in the various arthritides, but when he looks from the roentgenogram (first) to the patient he must be careful not to have already developed a fixed notion from the roentgenogram. I shall present a brief outline of the typical roentgenographic changes in the various forms of arthritis.

\section{Traumatic Arthritis}

A. Acute (accidental).

1. Mild trauma.

(a) May be periarticular swelling only.

2. Severe trauma.

(a) Some hypertrophic reaction may follow (baseball finger).

3. Very severe trauma.

(a) Hypertrophic reaction and even destructive changes may follow (hip-joint).

B. Chronic (recreational, postural, static).

1. Early.

(a) Usually negative findings.

(b) Perhaps small swelling (tennis wrist).

2. Late.

(a) Usually hypertrophic changes (prize-fighters). 


\section{Chemical Arthritis}

A. Serum sickness.

1. Usually negative findings.

2. Perhaps periarticular swelling.

B. Gout.

1. Acute.

(a) Early.

(1) Negative bone and cartilage.

(2) Periarticular swelling.

(b) Late.

(1) Periarticular swelling.

(2) Perhaps hypertrophy of bone.

2. Chronic.

(a) Early.

(1) Periarticular swelling.

- (2) Hypertrophy of bone.

(3) Some destruction of bone and cartilage.

(4) Small punched-out areas.

(b) Late.

(1) Periarticular swelling.

(2) Irregular, asymmetrical, dense areas (tophi) in soft tissue.

(3) Hypertrophy of bone.

(4) Destruction of bone and cartilage.

(5) Small and large areas of erosion.

\section{Infectious Arthritis}

A. Rheumatic fever.

1. Joints negative or periarticular swelling.

2. Never any reaction of bone or cartilage.

B. Psoriatic arthritis.

1. In general.

(a) Affects all joints.

(b) In no way diagnostically characteristic.

(c) In terminal finger-joints evidence is exactly like that of rheumatoid arthritis.

2. Early.

(a) Atrophy of bone.

(b) Periarticular swelling. 
3. Later.

(a) Destruction of cartilage.

(b) Slight hypertrophy of bone.

4. Latest.

(a) Changes of previous stages plus destruction of bone.

C. Gonorrhœal arthritis.

1. In general.

(a) Evidence same as in any phase of infectious arthritis, depending on stage and severity.

2. If arthralgia only.

(a) Negative findings.

3. If mild.

(a) Periarticular swelling.

(b) Perhaps some atrophy of bone.

4. If of moderate severity.

(a) Periarticular swelling.

(b) Atrophy of bone.

(c) Perhaps thickening of cartilage and slight hypertrophic arthritis.

5. If severe.

(a) Periarticular swelling.

(b) Destruction of cartilage and ankylosis of bone (early).

(c) Extent of destruction in marked relation to degree of osteoporosis and duration.

D. Arthritis of ulcerative colitis.

1. In general.

(a) Not diagnostically characteristic.

(b) Generally resembles mild rheumatoid arthritis (periarticular swelling, some atrophy of bone, perhaps thinning of cartilage).

2. If severe.

(a) Destruction of bone and cartilage.

(b) Some lipping of bone.

E. Rheumatoid (atrophic, proliferative, chronic infectious) arthritis.

1. Very early.

(a) Findings may be negative.

(b) Perhaps periarticular swelling. 
2. Moderately early.

(a) Perhaps atrophy of bone or osteoporosis.

3. Advancing.

(a) Thinning of cartilage.

4. Well advanced.

(a) Periarticular swelling.

(b) Atrophy of bone.

(c) Definite loss of cartilage.

5. Late.

(a) Moderate severity.

(1) Foregoing manifestations plus hypertrophy of bone. (N.B.-The hypertrophy affects weightbearing joints relatively early.)

(b) Severe.

(1) Little periarticular swelling.

(2) Severe atrophy of bone.

(3) Cartilage destroyed.

(4) Moderate lipping of bone.

(5) Severe destruction of bone.

(6) Severe disarrangement of joint.

(7) Ankylosis.

(8) Deformity.

F. Septic arthritis. (N.B.-Infection with staphylococci, gonococci, pneumococci, streptococci, Brucella.)

1. Early.

(a) Swelling of soft tissue.

2. Advanced.

(a) Swelling of soft tissue.

(b) Destruction.

3. Late.

(a) Destruction.

(b) Hypertrophic reactions.

(c) Ankylosis.

IV. Primary Osteo-arthritis (Hypertrophic, Senescent, Degenerative

A. In general. Arthritis)

1. Never ankylosis except as related to spurs.

B. Early and mild (finger).

1. Slight periarticular swelling.

2. Slight thinning of cartilage. 
C. Advancing.

1. Changes of early stage plus further destruction of cartilage.

2. Beginning of definite lipping of bone.

D. Late.

1. Slight (decreased) periarticular swelling.

2. Destruction of cartilage more or less complete.

3. Marked marginal lipping of bone.

4. One or both of the following: (1) subchondral bone; (2) areas of destruction and hypertrophy.

E. Later.

1. Deformation.

\section{Conclusions}

1. In arthritis, roentgenograms alone are rarely characteristic enough to be diagnostic of any specific ætiologic condition.

2. When the roentgenologist uses the terms "atrophic," and particularly "hypertrophic," he must be sure the clinician knows he is referring to the roentgenographic image and is not diagnosing a clinical syndrome-for example, "hypertrophic arthritis" (meaning senescent or primary osteo-arthritis). The two may or may not be synonymous. This may be important in medico-legal cases.

3. The roentgenographic "diagnosis" is not final, but always subject to correlation with the clinical data.

\section{RHEUMATIC FEVER AND NUTRITION*}

\section{BY JAMES F. RINEHART}

THE factors controlling the development of rheumatic fever afford an intriguing medical problem and one of major importance to public health. Most investigators have concerned themselves with the bacteriological aspect of the disease. That an infective agent is operative in the development of rheumatic fever can hardly be denied. The work of numerous students strongly implicates the hæmolytic streptococcus. ${ }^{1}$ The more recent work is particularly impressive. The common occurrence of respiratory infections with various strains of Group A hæmolytic streptococci preceding the onset of rheumatic fever

* This work has been aided by grants from the Christine Breon Fund for Medical Research and by donations from the California Fruit Growers Exchange and Hoffman-LaRoche, Inc. 